\title{
Phase Synchronization Is the Amplified Result by the Hilbert Transform
}

\author{
Ming-Chi Lu, ${ }^{1}$ Hsing-Chung Ho, ${ }^{2}$ Chen-An Chan, ${ }^{1}$ Chia-Ju Liu, ${ }^{2}$ \\ Jiann-Shing Lih, ${ }^{1}$ and Ming-Chung $\mathrm{Ho}^{1}$ \\ ${ }^{1}$ Department of Physics, National Kaohsiung Normal University, Kaohsiung 824, Taiwan \\ ${ }^{2}$ Graduate Institute of Science Education \& Environmental Education, National Kaohsiung Normal University, \\ Kaohsiung 824, Taiwan
}

Correspondence should be addressed to Ming-Chung Ho; t1603@nknucc.nknu.edu.tw

Received 30 September 2014; Accepted 26 December 2014

Academic Editor: Mo Li

Copyright $\odot 2015$ Ming-Chi Lu et al. This is an open access article distributed under the Creative Commons Attribution License, which permits unrestricted use, distribution, and reproduction in any medium, provided the original work is properly cited.

We investigate the interplay between phase synchronization and amplitude synchronization in nonlinear dynamical systems. It is numerically found that phase synchronization intends to be established earlier than amplitude synchronization. Nevertheless, amplitude synchronization (or the state with large correlation between the amplitudes) is crucial for the maintenance of a high correlation between two time series. A breakdown of high correlation in amplitudes will lead to a desynchronization of two time series. It is shown that these unique features are caused essentially by the Hilbert transform. This leads to a deep concern and criticism on the current usage of phase synchronization.

\section{Introduction}

Synchronization of chaotic systems has been an important area in nonlinear dynamics [1]. "Synchronization" is defined as a complete coincidence of two variables (or time series) that are belonging to different systems [2] while the appearance of some functional relations between two variables is termed as "generalized synchronization" [3, 4]. Instead of focusing on the synchronization of time series, Rosenblum et al. introduced the concept of phase synchronization to describe how the coupled chaotic oscillators could present a nearly perfect locking of phase, whereas the amplitude remained chaotic [5]. In [5], the phase of a time series was defined based on the Hilbert transform [6]. This definition is also very attractive in characterization of chaos [7]. In a more explicit form, the Hilbert transform of a time series $x(t)$ follows:

$$
\widehat{x}(t)=\frac{1}{\pi} \mathrm{P} \cdot \mathrm{V} \cdot \int_{-\infty}^{\infty} \frac{x(\tau)}{t-\tau} d \tau
$$

where P.V. means the Cauchy principal value for the integral. Thus, a new complex quantity $\psi(t)$ can be introduced; that is,

$$
\psi(t)=x(t)+i \widehat{x}(t)=A_{H}(t) e^{i \theta_{H}(t)},
$$

where $A_{H}(t)$ is the phase and $\theta_{H}(t)$ is the amplitude $[5,7]$ and they form a conjugate pair. One can also define the phase angle to be the projection of phase point on the $x-y$ plane with the phase angle $\theta_{T}(t)=\tan ^{-1}(y / x)$ and the amplitude $A_{T}(t)=\sqrt{x^{2}+y^{2}}$. Alternatively by using the Poincare section, one can also choose a phase:

$$
\theta_{P}(t)=2 \pi \frac{t-t_{n}}{t_{n+1}-t_{n}}+2 n \pi,
$$

where $t_{n}<t<t_{n+1}$ and $t_{n}$ denotes the $n$th crossing, but there is no conjugate amplitude for $\theta_{P}(t)$. In this comment, we reexamine the virtue of using the phase variable as the indicator of synchronization of the time series. We believe that phase synchronization may not be a good tracer to the actual synchronization of the time series. Then our work will 
explain the onset mechanism of phase synchronization and it leads to a deep concern on the current usage of phase synchronization.

\section{Give an Example of Coupled Rossler Model}

Let us recalculate the same coupled Rossler model as in [5] to explore the synchronization. The model follows:

$$
\begin{aligned}
& \dot{x}_{1,2}=-\omega_{1,2} y_{1,2}-z_{1,2}+C\left(x_{2,1}-x_{1,2}\right), \\
& \dot{y}_{1,2}=\omega_{1,2} x_{1,2}+0.15 y_{1,2}, \\
& \dot{z}_{1,2}=0.2+z_{1,2}\left(x_{1,2}-10\right),
\end{aligned}
$$

where $C$ is the strength of coupling, $\omega_{1,2}=1 \pm \Delta \omega$, and $\Delta \omega=0.015$ which indicates that there is a frequency mismatch between two oscillators. Because of finite frequency mismatch, there is no exact synchronization in time series. Synchronization can be found for $C$ not only in the range $[0,1]$, as reported in [5], region I, but also in [2.9, 3.45], region II. Thus, in such a case, "synchronization" only implies a high correlation between two variables, such as time series. By using the Hilbert transform, the variables $x_{i}$ have the phase $\theta_{i}$ and the amplitude $A_{i}(i=1,2)$. It has been reported that $\theta_{1}$ and $\theta_{2}$ move together and get nearly synchronized, while $A_{1}$ and $A_{2}$ remain irregular and unrelated in a range of small $C$ [5]. Since then, cited and extended works have been expanded dramatically [8-19]. However, it should be emphasized that as phase is introduced, the influence of its counterpart, that is, amplitude, and their interplay should not be ignored. Unfortunately, although the concept of phase synchronization has been extensively addressed, the correlation between phase synchronization and amplitude synchronization remains to be clarified.

\section{Results and Discussion}

Let us use a common measure, the mean square error, to quantify the degree of synchronization. For two time series, $x_{1}$ and $x_{2}$, the mean square error is defined as

$$
\sigma_{x}=\frac{1}{T} \int_{0}^{T}\left[x_{1}(t)-x_{2}(t)\right]^{2} d t
$$

where the integration time $T$ should be sufficiently long. For the phase and the amplitude deduced by the Hilbert transform, we denote their mean square errors to be $H_{\theta}$ and $H_{A}$. For phase angle defined by projection (here $x_{i}$ and $y_{i}$ of the Rossler model), the corresponding mean square errors are $T_{\theta}$ and $T_{A}$, while in the case of the Poincare section (here a typical section in the $x_{i}-y_{i}$ plane) we use $P_{\theta}$ to denote it. Obviously, to be good indicators of the synchronization, variations of these quantities should faithfully reflect the true status of the coupled chaotic oscillators. As shown in Figure 1, where the values of the mean square errors in region I are plotted, the mean square errors of the phase variables are insensitive to the true state of synchronization in time series. In contrast, the mean square errors of the conjugate amplitudes reflect more faithfully to the status of oscillators

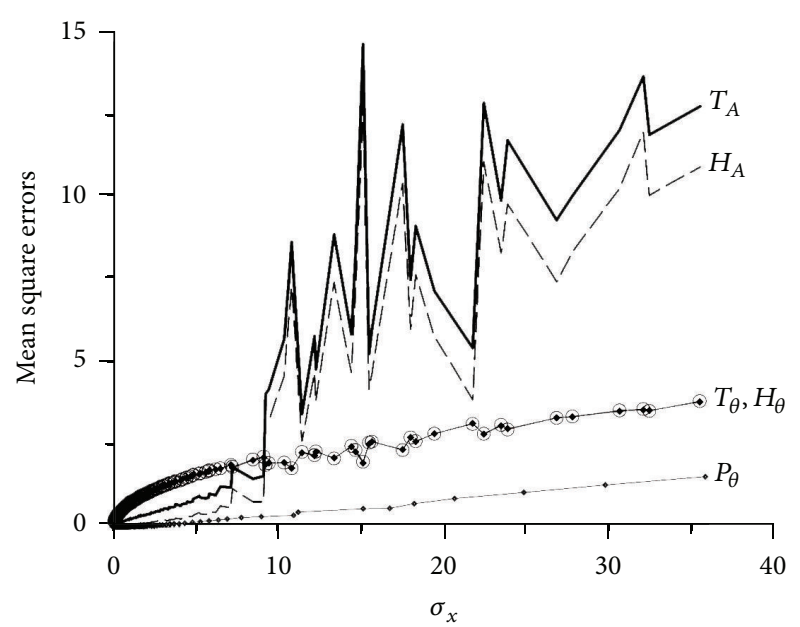

FIGURE 1: The mean square error (mse) diagram of the coupled Rossler model. $\sigma_{x}$ is the mse of the time series. $H_{\theta}, T_{\theta}$ and $P_{\theta}$ are the mse for the phase variables while $H_{A}$ and $T_{A}$ are for the amplitudes.

in this case. Similar feature can also be found in region II as well as for different coupled oscillators.

\section{Conclusion}

The result shows that phase synchronization will intend to be established earlier than amplitude synchronization under the Hilbert transform. This unique feature is novel, but it is caused by the Hilbert transform. The transformation on the phase part is nonlinear. This nonlinear transform is the generic mechanism for the novel features that have been reported on the phase synchronization [5, 6, 8-19]. Therefore, we believe the role of "phase" and phase synchronization may not be a good indicator to the true synchronization in the time series. The relevance of phase variables to the synchronization of the original variables in nonlinear dynamical systems seems to be a mathematical consequence of the transform one used. Thus, the works on phase synchronization $[6,13-19]$ may be worthwhile to be reconsidered.

\section{Conflict of Interests}

There is no conflict of interests related to this paper.

\section{Acknowledgment}

The authors would like to thank the National Science Council, Taiwan, for financially supporting this research under contract no. NSC 102-2112-M-017-002-.

\section{References}

[1] L. M. Pecora and T. L. Carroll, "Synchronization in chaotic systems," Physical Review Letters, vol. 64, pp. 821-824, 1990.

[2] L. Kocarev and U. Parlitz, "General approach for chaotic synchronization with applications to communication," Physical Review Letters, vol. 74, no. 25, pp. 5028-5031, 1995. 
[3] N. F. Rulkov, M. M. Sushchik, L. S. Tsimring, and H. D. I. Abarbanel, "Generalized synchronization of chaos in directionally coupled chaotic systems," Physical Review E, vol. 51, no. 2, pp. 980-994, 1995.

[4] L. Kocarev and U. Parlitz, "Generalized synchronization, predictability, and equivalence of unidirectionally coupled dynamical systems," Physical Review Letters, vol. 76, no. 11, pp. 1816$1819,1996$.

[5] M. G. Rosenblum, A. S. Pikovsky, and J. Kurths, "Phase synchronization of chaotic oscillators," Physical Review Letters, vol. 76, no. 11, pp. 1804-1807, 1996.

[6] M. G. Rosenblum, A. S. Pikovsky, and J. Kurths, "From phase to lag synchronization in coupled chaotic oscillators," Physical Review Letters, vol. 78, no. 22, pp. 4193-4196, 1997.

[7] T. Yalçınkaya and Y.-C. Lai, "Phase characterization of chaos," Physical Review Letters, vol. 79, no. 20, pp. 3885-3888, 1997.

[8] A. S. Pikovsky, M. G. Rosenblum, G. V. Osipov, and J. Kurths, "Phase synchronization of chaotic oscillators by external driving," Physica D, vol. 104, no. 3-4, pp. 219-238, 1997.

[9] P. Tass, J. Kurths, M. G. Rosenblum, G. Gauasti, and H. Hefer, "Delay-induced transitions in visually guided movements," Physical Review E, vol. 54, Article ID R2224, 1996.

[10] D. Y. Tang and N. R. Heckenberg, "Synchronization of mutually coupled chaotic systems," Physical Review E: Statistical Physics, Plasmas, Fluids, and Related Interdisciplinary Topics, vol. 55, no. 6, pp. 6618-6623, 1997.

[11] P. S. Landa, A. A. Zaikin, M. G. Rosenblum, and J. Kurths, "Onoff intemittency phenomena in a pendulum with a randomly vibrating suspension axis," Chaos, Solitons \& Fractals, vol. 9, no. 1-2, pp. 157-169, 1998.

[12] M. Palus, "Detecting phase synchronization in noisy systems," Physics Letters A, vol. 235, no. 4, pp. 341-351, 1997.

[13] T. F. Hsu, K. H. Jao, and Y. C. Hung, "Phase synchronization in a two-mode solid state laser: periodic modulations with the second relaxation oscillation frequency of the laser output," Physics Letters A, vol. 378, no. 44, pp. 3269-3273, 2014.

[14] Y. Kawamura, "Collective phase dynamics of globally coupled oscillators: noise-induced anti-phase synchronization," Physica D, vol. 270, pp. 20-29, 2014.

[15] K. J. Lee, Y. Kwak, and T. K. Lin, "Phase jumps near a phase synchronization transition in systems of two coupled chaotic oscillators," Physical Review Letters, vol. 81, no. 2, pp. 321-324, 1998.

[16] E. Rosa Jr., E. Ott, and M. H. Hess, “Transition to phase synchronization of chaos," Physical Review Letters, vol. 80, no. 8, pp. 1642-1645, 1998.

[17] R. C. Elson, A. I. Selverston, R. Huerta, N. F. Rulkov, M. I. Rabinovich, and H. D. I. Abarbanel, "Synchronous behavior of two coupled biological neurons," Physical Review Letters, vol. 81, no. 25, pp. 5692-5695, 1998.

[18] R. Ma, G. S. Klindt, I. H. Riedel-Kruse, F. Jülicher, and B. M. Friedrich, "Active phase and amplitude fluctuations of flagellar beating," Physical Review Letters, vol. 113, Article ID 048101, 2014.

[19] F. Pessacg, A. Taitz, G. Patterson, P. Fierens, and D. Grosz, "Experimental demonstration of a noise-tunable delay line with applications to phase synchronization," Communications in Nonlinear Science and Numerical Simulation, vol. 22, no. 1-3, pp. 872-876, 2015. 


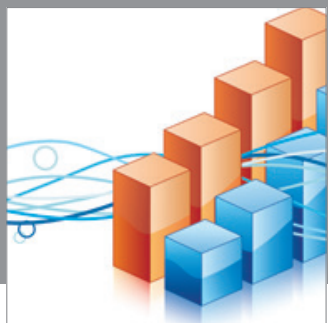

Advances in

Operations Research

mansans

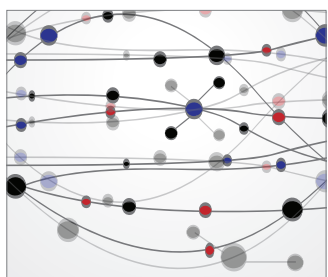

The Scientific World Journal
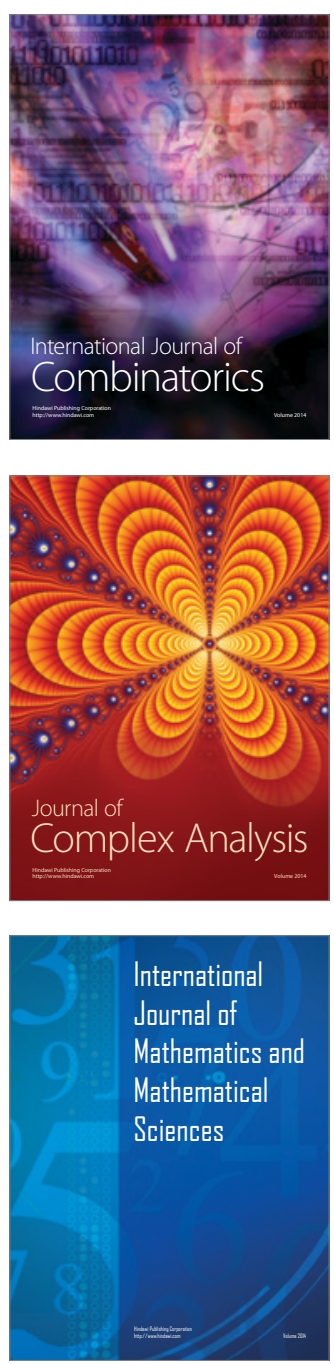
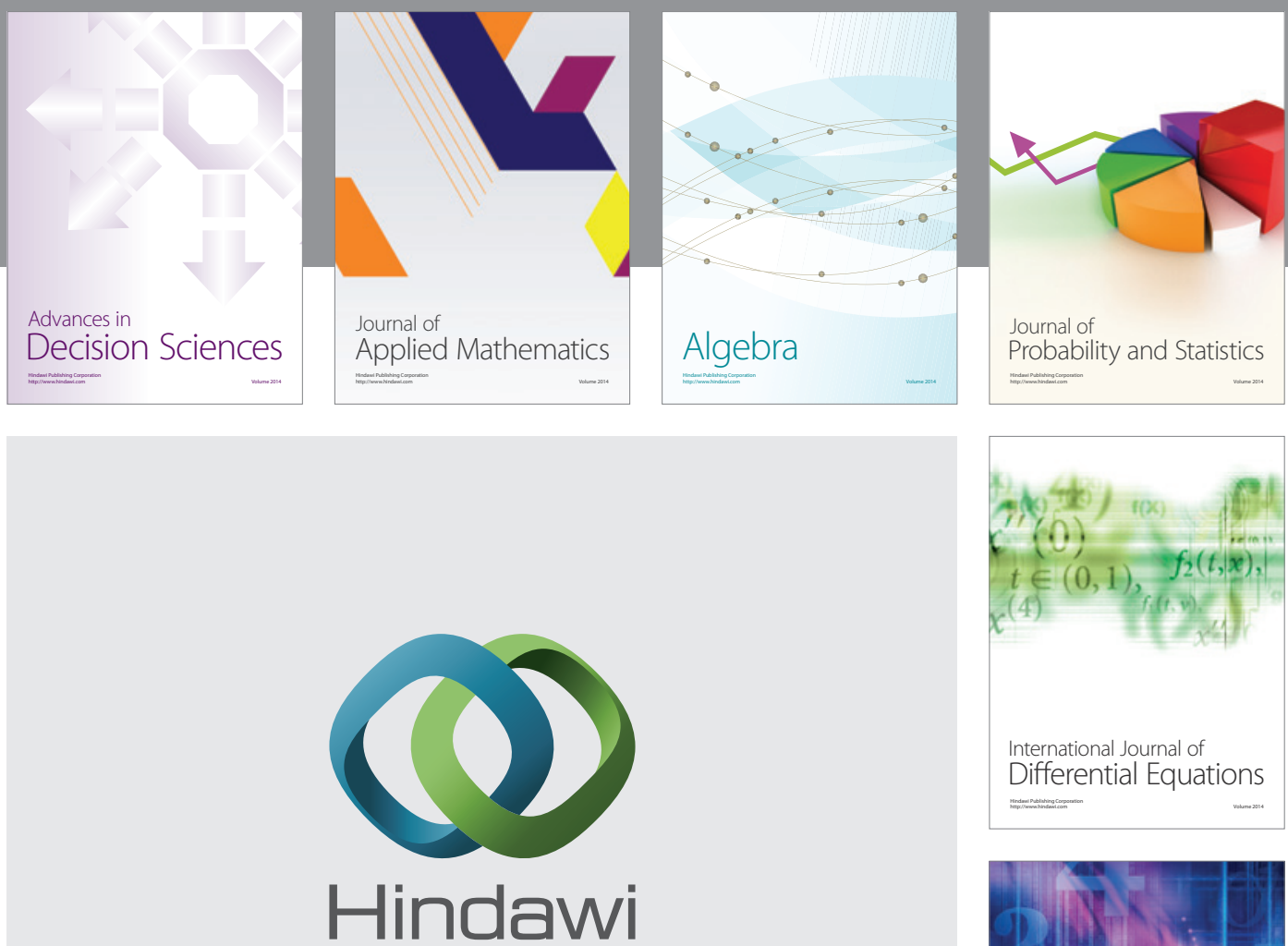

Submit your manuscripts at http://www.hindawi.com
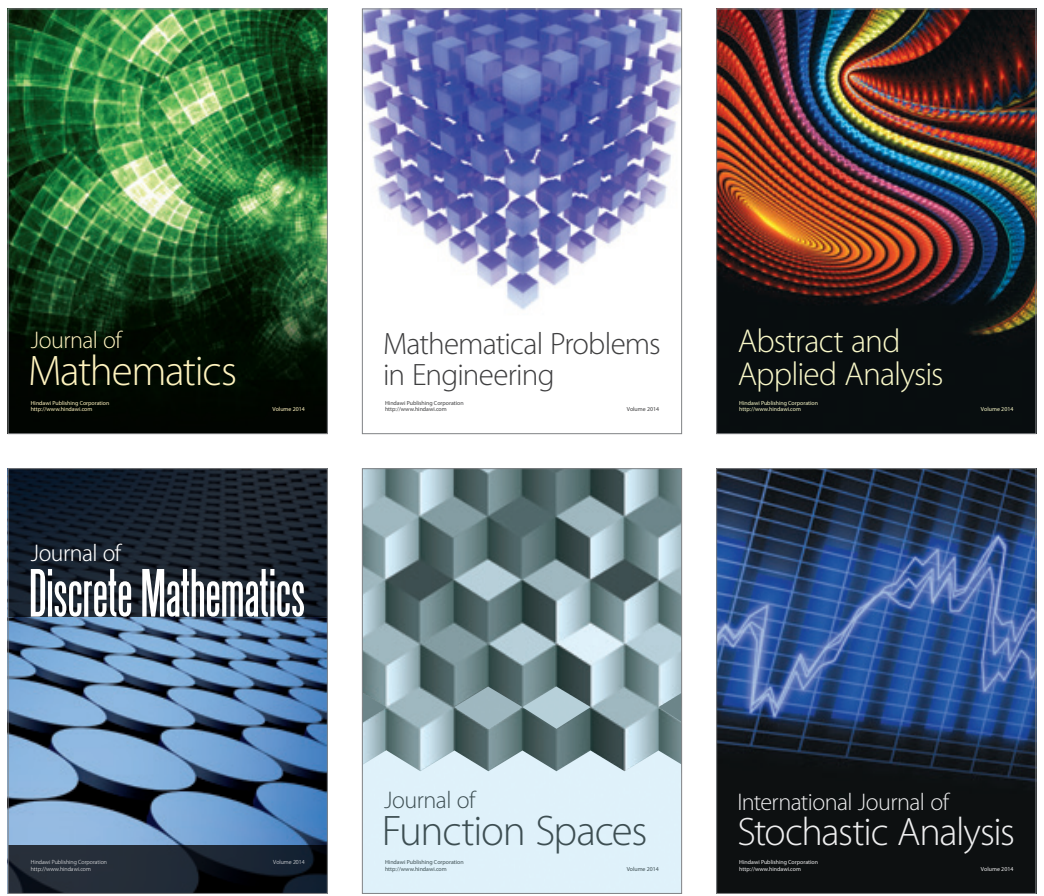

Journal of

Function Spaces

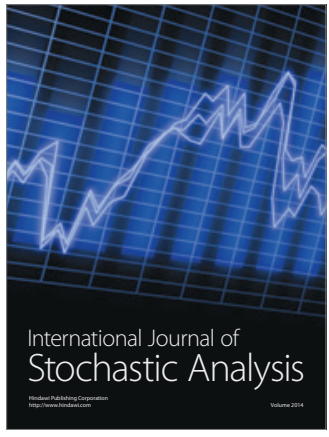

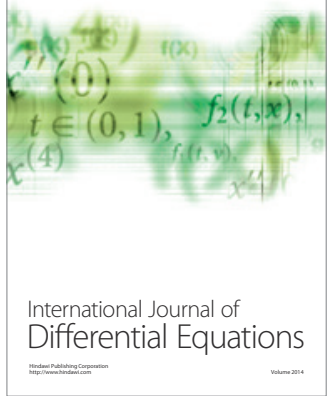
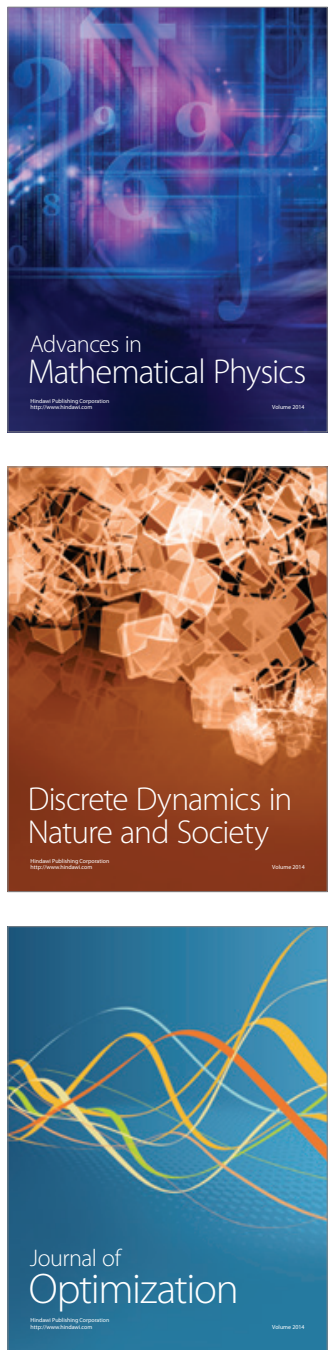